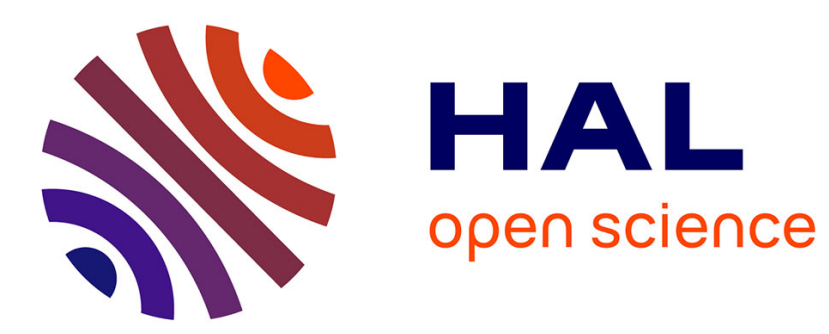

\title{
A Timoshenko finite element straight beam with internal degrees of freedom
}

Denis Caillerie, Panagiotis Kotronis, Robert Cybulski

\section{To cite this version:}

Denis Caillerie, Panagiotis Kotronis, Robert Cybulski. A Timoshenko finite element straight beam with internal degrees of freedom. International Journal for Numerical and Analytical Methods in Geomechanics, 2015, 39 (16), pp.1753-1773. 10.1002/nag.2367 . hal-01161516

\author{
HAL Id: hal-01161516 \\ https://hal.science/hal-01161516
}

Submitted on 14 Jan 2020

HAL is a multi-disciplinary open access archive for the deposit and dissemination of scientific research documents, whether they are published or not. The documents may come from teaching and research institutions in France or abroad, or from public or private research centers.
L'archive ouverte pluridisciplinaire HAL, est destinée au dépôt et à la diffusion de documents scientifiques de niveau recherche, publiés ou non, émanant des établissements d'enseignement et de recherche français ou étrangers, des laboratoires publics ou privés. 


\title{
A Timoshenko finite element straight beam with internal degrees of freedom
}

\author{
D. Caillerie ${ }^{\mathrm{a}}$, P. Kotronis ${ }^{\mathrm{b}}$, R. Cybulski ${ }^{\mathrm{c}}$ \\ ${ }^{a}$ Laboratoire 3S-R (Sols, Solides, Structures-Risques) INPG/UJF/CNRS UMR 5521 \\ Domaine Universitaire, BP 53, 38041, Grenoble, cedex 9, France \\ ${ }^{b}$ LUNAM Université, Ecole Centrale de Nantes, Université de Nantes, CNRS UMR 6183, GeM (Institut de Recherche en Génie Civil et Mécanique) \\ 1 rue de la Nö̈, BP 92101, 44321, Nantes, cedex 3, France \\ Panagiotis.Kotronis@ec-nantes.fr (correspondingauthor) \\ ${ }^{c}$ Silesian University of Technology, Theory of Building Structures Department, Akademicka 5, 44-100 Gliwice, Poland
}

\begin{abstract}
We present hereafter the formulation of a Timoshenko finite element straight beam with internal degrees of freedom, suitable for non linear material problems in geomechanics (e.g. beam type structures, deep pile foundations ...) Cubic shape functions are used for the transverse displacements and quadratic for the rotations. The element is free of shear locking and we prove that one element is able to predict the exact tip displacements for any complex distributed loadings and any suitable boundary conditions. After the presentation of the virtual power and the weak form formulations, the construction of the elementary stiffness matrix is detailed. The analytical results of the static condensation method are provided. It is also proven that the element introduced by Friedman and Kosmatka in [11], with shape functions depending on material properties, is derived from the new beam. Validation is provided using linear and material non linear applications (reinforced concrete column under cyclic loading) in the context of a multifiber beam formulation.
\end{abstract}

Keywords: beam, shear locking, Timoshenko, multifiber

\section{Introduction}

In [11], Friedman and Kosmatka have introduced a very efficient two node Timoshenko finite element beam using cubic and quadratic Lagrangian polynomials for the transverse displacements and rotations respectively. The polynomials are made interdependent by requiring them to satisfy the two homogeneous differential equations associated with Timoshneko's beam theory. The resulting stiffness matrix is exactly integrated and the element is free of shear locking. The authors numerically verified that one element is able to predict the exact tip displacement of a cantilever Timoshenko beam subjected to either an applied transverse tip load, a uniform load or a linear varying distributed load.

Although this element is widely used, see for example [5], its domain of application is limited because of the nature of its shape functions that depend on material properties. We propose hereafter an improved Timoshenko finite element beam with three (3) in 2D or six (6) in 3D internal degrees of freedom and similar numerical capacities. Cubic shape functions are used for the transverse displacements and quadratic for the rotations. The shape functions are independent on material properties. The new element, called hereafter FCQ Timoshenko beam for Full Cubic Quadratic, is free of shear locking and one element is able to predict the exact tip displacements for any complex distributed loadings and any suitable boundary conditions. That element turns out to yield the same nodal degrees of freedom as those of the element presented in [11] but the interpolations of the transverse displacements and rotation functions are different.

In section 2 we introduce the notations, the balance and constitutive equations and the dimensionless variables of the problem. In sections 3 and 4 we present the virtual power and weak form formulations and the way to obtain the analytical solution 
of a 2D cantilever Timoshenko beam subjected to distributed loadings in terms of loads (forces and moments) and of displacements and rotations at both ends. Sections 5 and 6 deal with the construction of the elementary stiffness matrix of the FCQ element and section 7 with the calculation of the internal degrees of freedom and the reduced stiffness matrix using the static condensation method. In section 8 we prove analytically that the element introduced in [11] can be derived from the FCQ Timoshenko beam. Section 9 contains the analytical proof that the new element predicts the exact tip displacements for any complex distributed loadings and any suitable boundary conditions. Finally, section 10 provides validation of the FCQ Timoshenko beam under static and cyclic loadings. More specifically, we treat the examples of:

- A cantilever Timoshenko beam subjected to a complex distributed transverse load (polynomial function).

- A cantilever Timoshenko beam subjected to a constant distributed transverse load and a linear distributed bending moment.

- A reinforced concrete column submitted to a cyclic load. For this example, the behaviour of the materials is considered non elastic and the column is simulated using multifiber Timoshenko FCQ beams.

\section{Balance and constitutive equations}

\subsection{Notations}

Let's consider a Timoshenko beam of length $l$, having an homogeneous cross-section of area $A$ and of moment of inertia $I$. $E$ and $G$ are the Young and shear moduli of the elastic material of the beam. $\kappa$ is the shear coefficient [7] involved in the constitutive equation for the shear force. A Cartesian coordinate system $(\hat{x}, \hat{y}, \hat{z})$ is defined on the beam where the $\hat{x}$ axis is coincident with the centroidal axis (Fig. 1).

For the sake of simplicity, the study is restricted to the flexural static behaviour in the $\hat{x}-\hat{z}$ plane. $\hat{w}$ is the transverse displacement according to the $\hat{z}$ axis and $\hat{\theta}$ the rotation of the crosssection about the $\hat{y}$ axis. Let $\hat{T}$ and $\hat{M}$ denote the shear force and

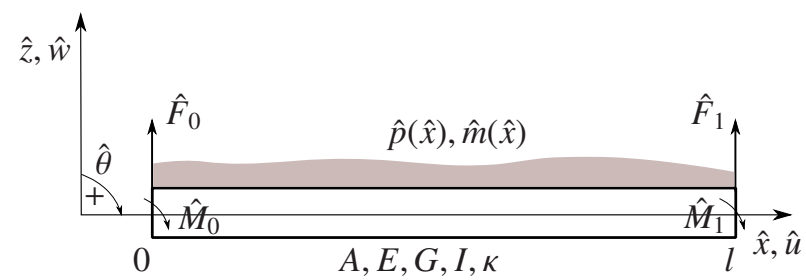

Figure 1: 2D Timoshenko beam and applied loads.

the bending moment along the beam. The Timoshenko beam can be subjected to a consistent (see Section 2.2) combination of a distributed load $\hat{p}(\hat{x})$, a distributed moment $\hat{m}(\hat{x})$, applied forces and moments $\hat{F}_{0}$ and $\hat{M}_{0}$ at $\hat{x}=0$ and $\hat{F}_{1}$ and $\hat{M}_{1}$ at $\hat{x}=l$, applied displacements and rotations $\hat{w}_{0}, \hat{\theta}_{0}$ at $\hat{x}=0$ and $\hat{w}_{1}, \hat{\theta}_{1}$ at $\hat{x}=l$. The equilibrium in a section of infinitesimal length $d \hat{x}$ is shown in Fig. 2.

$$
\overbrace{\hat{M}} \hat{T}\left|\sum_{d \hat{x}}^{\hat{p}}\right| \hat{T}+d \hat{T}\rangle^{\hat{m}} \hat{M}+d \hat{M}
$$

Figure 2: Equilibrium in a section of infinitesimal length.

In order to simplify the writing of the equations, we use dimensionless functions and variables defined as:

$$
\begin{aligned}
& x=\frac{\hat{x}}{l} \quad(0 \leq x \leq 1), \quad w=\frac{\hat{w}}{l}, \quad \theta=\hat{\theta}, \\
& w_{i}=\frac{\hat{w}_{i}}{l}, \quad \theta_{i}=\hat{\theta}_{i}, \quad(i=0,1),
\end{aligned}
$$

$$
\begin{aligned}
& M=\frac{l}{E I} \hat{M}, \quad T=\frac{l^{2}}{E I} \hat{T}, \quad p=\frac{l^{3}}{E I} \hat{p}, \quad m=\frac{l^{2}}{E I} \hat{m}, \\
& F_{i}=\frac{l^{2}}{E I} \hat{F}_{i}, \quad M_{i}=\frac{l}{E I} \hat{M}_{i}, \quad(i=0,1) .
\end{aligned}
$$

\subsection{Balance and constitutive equations}

The classical balance equations expressed using the dimensionless quantities of Eqs. 1 and 2 take the following form (see Fig. 2):

$$
\begin{aligned}
& \frac{d T}{d x}+p=0, \\
& \frac{d M}{d x}-T+m=0 .
\end{aligned}
$$


According to the Timoshenko beam theory (sections remain plane but not perpendicular to the neutral axis) and the definition of the dimensionless functions and variables of Eqs. 1 and 2 , the classical constitutive equations take the form:

$$
\begin{aligned}
M & =\frac{d \theta}{d x}, \\
T & =\frac{1}{e}\left(\theta+\frac{d w}{d x}\right),
\end{aligned}
$$

where:

$$
\frac{1}{e}=\frac{\kappa G A l^{2}}{E I} .
$$

Remarks:

- The boundary conditions $\left(w_{0}, \theta_{0}, w_{1}, \theta_{1}, F_{0}, M_{0}, F_{1}, M_{1}\right)$ have to be consistent, in other words they cannot be set independently (see also the following remark on global equilibrium or the exact solution in Eq. 14). Only some of the boundary conditions imposed to the beam are known. For instance, for the case of a cantilever beam with tip loads, $w_{0}$ and $\theta_{0}$ are set to $0, F_{1}$ and $M_{1}$ are given and $w_{1}, \theta_{1}, F_{0}$ and $M_{0}$ are unknown.

- Following Figs. 1 and 2, the kinematic as well as the load boundary equations read:

$$
\begin{array}{ll}
w(0)=w_{0}, & w(1)=w_{1}, \\
\theta(0)=\theta_{0}, & \theta(1)=\theta_{1}, \\
T(0)=-F_{0}, & T(1)=F_{1}, \\
M(0)=-M_{0}, & M(1)=M_{1} .
\end{array}
$$

- The external forces and moments have to satisfy the global equilibrium equations:

$$
\begin{aligned}
& F_{0}+F_{1}+\int_{0}^{1} p d x=0 \\
& M_{0}+M_{1}-F_{1}+\int_{0}^{1}(m-x p) d x=0 .
\end{aligned}
$$

- The parameter e defined at Eq. 5 is of the order of the aspect ratio (slenderness) of the beam. Indeed, $E$ and $G$ are of the same order and $\kappa$ is generally taken of order 0 with respect to the aspect ratio of the beam. For a slender beam, $e$ is thus small and consequently $\theta+\frac{d w}{d x}$ is also small. In other words, in that case the Timoshenko beam is almost a Navier Bernoulli beam (for which $\theta+\frac{d w}{d x}=0$ ). This provides a first explanation why locking can occur in finite element simulations of bending of slender Timoshenko beams, the Navier Bernoulli condition requiring too many degrees of freedom to be fulfilled (see also [3], [9] and [13]).

\section{Weak formulation}

The virtual power formulation classically reads (the symbol * denoting a virtual field):

$$
\begin{aligned}
& \forall\left(w^{*}, \theta^{*}\right),-\int_{0}^{1}\left(M \frac{d \theta^{*}}{d x}+T\left(\theta^{*}+\frac{d w^{*}}{d x}\right)\right) d x \\
& +\int_{0}^{1}\left(p w^{*}+m \theta^{*}\right) d x \\
& +F_{0} w_{0}^{*}+M_{0} \theta_{0}^{*}+F_{1} w_{1}^{*}+M_{1} \theta_{1}^{*}=0 .
\end{aligned}
$$

Substituting in Eq. 8 the constitutive laws (Eqs. 4 and 5) and the boundary conditions (Eqs. 6) we get the variational equation involved in any weak formulation of the problem:

$$
\begin{aligned}
& \forall\left(w^{*}, \theta^{*}\right), a\left((w, \theta),\left(w^{*}, \theta^{*}\right)\right)=L\left(w^{*}, \theta^{*}\right) \\
& +F_{0} w_{0}^{*}+M_{0} \theta_{0}^{*}+F_{1} w_{1}^{*}+M_{1} \theta_{1}^{*}
\end{aligned}
$$

where the bilinear and linear forms $a\left((w, \theta),\left(w^{*}, \theta^{*}\right)\right)$ and $L\left(w^{*}, \theta^{*}\right)$ are defined by:

$$
\begin{aligned}
& a\left((w, \theta),\left(w^{*}, \theta^{*}\right)\right)= \\
& \int_{0}^{1}\left(\frac{d \theta}{d x} \frac{d \theta^{*}}{d x}+\frac{1}{e}\left(\theta+\frac{d w}{d x}\right)\left(\theta^{*}+\frac{d w^{*}}{d x}\right)\right) d x, \\
& L\left(w^{*}, \theta^{*}\right)=\int_{0}^{1}\left(p w^{*}+m \theta^{*}\right) d x .
\end{aligned}
$$

\section{Analytical solutions}

The general solution of Eqs. 3, taking into account the boundary condtions (Eq. 6) reads:

$$
\begin{aligned}
T^{e x a} & =-p_{1}-F_{0}, \\
M^{\text {exa }} & =-p_{2}-m_{1}-F_{0} x-M_{0},
\end{aligned}
$$


for the shear force and the moment and:

$$
\begin{aligned}
w^{e x a} & =-e p_{2}+p_{4}+m_{3}-F_{0}\left(e x-\frac{x^{3}}{6}\right) \\
& +M_{0} \frac{x^{2}}{2}-\theta_{0} x+w_{0}, \\
\theta^{e x a} & =-p_{3}-m_{2}-F_{0} \frac{x^{2}}{2}-M_{0} x+\theta_{0},
\end{aligned}
$$

for the the displacement and the rotation, where the successive primitives $p_{k}(x)$ and $m_{k}(x)$ of $p$ and $m$ are defined such that:

$$
\begin{array}{ll}
p_{0}(x)=p(x), & m_{0}(x)=m(x), \\
p_{k}(x)=\int_{0}^{x} p_{k-1}(y) \mathrm{d} y, & m_{k}(x)=\int_{0}^{x} m_{k-1}(y) \mathrm{d} y .
\end{array}
$$

Eqs. 13 can also be written in terms of $F_{1}$ and $M_{1}$ instead of $F_{0}$ and $M_{0}$ :

$$
\begin{aligned}
w^{e x a} & =p_{1}(1)\left(-\frac{x^{3}}{6}+\frac{x^{2}}{2}+e x\right)-e p_{2}-\left(p_{2}(1)+m_{1}(1)\right) \frac{x^{2}}{2} \\
& +p_{4}+m_{3}+F_{1}\left(-\frac{x^{3}}{6}+\frac{x^{2}}{2}+e x\right)-M_{1} \frac{x^{2}}{2}-\theta_{0} x+w_{0} \\
\theta^{e x a} & =p_{1}(1)\left(\frac{x^{2}}{2}-x\right)+\left(p_{2}(1)+m_{1}(1)\right) x-p_{3}-m_{2} \\
& +F_{1}\left(\frac{x^{2}}{2}-x\right)+M_{1} x+\theta_{0}
\end{aligned}
$$

Remarks:

- Replacing the dimensionless variables with their counterparts provided in Eqs. 1 and 2, one finds the classical solutions of the problem (expressed in dimensional variables).

- For $e=0$ Eqs. $13 a$ and $14 b$ provide the analytical transverse displacement for the Navier Bernoulli beam.

Let's now consider the case of a Timoshenko beam submitted to displacements $\left(w_{0}, w_{1}\right)$ and rotations $\left(\theta_{0}, \theta_{1}\right)$ at both ends $\left(x_{0}=0, x_{1}=1\right)$.

Taken at $x=1$, Eqs. 12 and 13 yield a linear system enabling to determine $T_{0}, M_{0}, T_{1}$ and $M_{1}$ in terms of the displacements and rotations $w_{0}, \theta_{0}, w_{1}$ and $\theta_{1}$. Written in matrix format, the result reads:

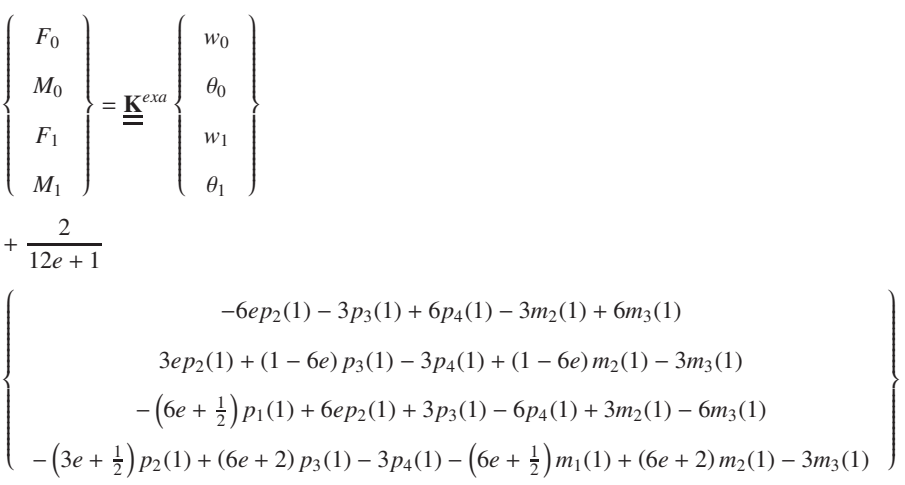

where the stiffness matrix is:

$$
\underline{\underline{\mathbf{K}}}^{\text {exa }}=\frac{2}{12 e+1}\left(\begin{array}{cccc}
6 & -3 & -6 & -3 \\
-3 & 6 e+2 & 3 & -6 e+1 \\
-6 & 3 & 6 & 3 \\
-3 & -6 e+1 & 3 & 6 e+2
\end{array}\right) .
$$

Remark: Eq. 15 can be seen as a compatibility condition between the boundary values $w_{0}, \theta_{0}, w_{1}, \theta_{1}, T_{0}, M_{0}, T_{1}$ and $M_{1}$.

\section{Finite element technology}

In this section we present a brief introduction on the finite element technology for a Timoshenko beam using the method of the parent element (which in this case is not isoparametric). Only the bending behaviour is considered in the developments presented hereafter. The following steps are performed:

- The domain where the problem is set, that is the segment $[0,1]$, is discretised into $N$ elements $E_{n}=\left[x_{n}, x_{n+1}\right]$ of length $\ell_{n}=x_{n}-x_{n+1},(1 \leq n \leq N)$, see figure 3 .

- The integrals of Eqs. 10 and 11 are written as sums of integrals over all the elements $E_{n}$.

- A change of variables for each element $E_{n}$ to the parent element $S_{R}=[-1,1]$ is carried out, see figure 4 .

Remark: The introduction of the parent element simplifies the numerical integration as well as the analytical calculation of the integrals. 


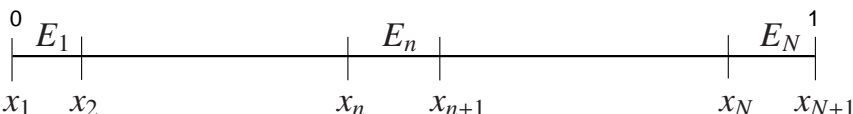

Figure 3: Discretised segment

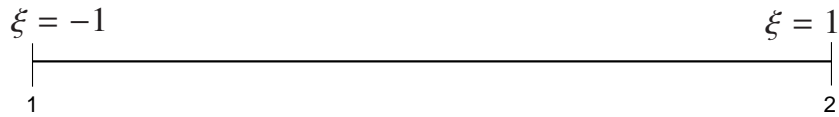

Figure 4: Parent element $S_{R}$.

The change of variables from $S_{R}$ to $E_{n}$ is given by the function $\phi_{n}(\xi)$ :

$$
\xi \leftrightarrow x=\phi_{n}(\xi)=N_{R}^{1}(\xi) x_{n}+N_{R}^{2}(\xi) x_{n+1},
$$

where the interpolation functions $N_{R}^{1}$ and $N_{R}^{2}$ are defined on the parent element $S_{R}$ as follows:

$$
N_{R}^{1}(\xi)=\frac{1}{2}(1-\xi), \quad N_{R}^{2}(\xi)=\frac{1}{2}(1+\xi)
$$

and so:

$$
\phi_{n}(\xi)=\frac{1}{2}\left(x_{n}+x_{n+1}\right)+\frac{\xi}{2} \ell_{n} .
$$

Therefore, the expressions 10 and 11 read:

$$
a\left((w, \theta),\left(w^{*}, \theta^{*}\right)\right)=\sum_{n=1}^{N} a_{n}\left(\left(\widetilde{w}_{n}, \widetilde{\theta}_{n}\right),\left(\widetilde{w_{n}^{*}}, \widetilde{\theta_{n}^{*}}\right)\right)
$$

and

$$
L\left(w^{*}, \theta^{*}\right)=\sum_{n=1}^{N} L_{n}\left(\widetilde{w_{n}^{*}}, \widetilde{\theta_{n}^{*}}\right),
$$

where

$$
\begin{aligned}
& a_{n}\left(\left(\widetilde{w}_{n}, \widetilde{\theta}_{n}\right),\left(\widetilde{w_{n}^{*}}, \widetilde{\theta}_{n}^{*}\right)\right)=\frac{2}{\ell_{n}} \int_{-1}^{1} \frac{d \widetilde{\theta}_{n}}{d \xi} \frac{d \widetilde{\theta}_{n}^{*}}{d \xi} d \xi \\
& +\frac{\ell_{n}}{2 e} \int_{-1}^{1}\left(\widetilde{\theta_{n}}+\frac{2}{\ell_{n}} \frac{d \widetilde{w}_{n}}{d \xi}\right)\left(\widetilde{\theta_{n}^{*}}+\frac{2}{\ell_{n}} \frac{d \widetilde{w}_{n}^{*}}{d \xi}\right) d \xi
\end{aligned}
$$

and

$$
L_{n}\left(\widetilde{w_{n}^{*}}, \widetilde{\theta_{n}^{*}}\right)=\frac{\ell_{n}}{2} \int_{-1}^{1}\left(\widetilde{p_{n}} \widetilde{w_{n}^{*}}+\widetilde{m_{n}} \widetilde{\theta_{n}^{*}}\right) d \xi
$$

In Eqs. 20, 21, 22 and 23 the tilted functions are defined according to the definition that follows: Let's $h(x)$ be a function defined on the discretised segment $[0,1]$, the functions $\widetilde{h}_{n}(\xi)$ are defined on the parent element $S_{R}$ as:

$$
\widetilde{h}_{n}(\xi)=h\left(\phi_{n}(\xi)\right)
$$

where $\phi_{n}$ is defined in Eq. 19. It's also obvious that with $N$ functions $\widetilde{h}_{n}(\xi)$ it's possible to uniquely define a function $h(x)$ on $[0,1]$ satisfying Eq. 24.

Up to now no approximation has been made. We have just performed a change of the unknowns of the problem of Eq. 9 which are no more the functions $w(x)$ and $\theta(x)$ defined on $[0,1]$ but the functions $\widetilde{w}_{n}(\xi)$ and $\widetilde{\theta}_{n}(\xi)$ defined on the parent element $S_{R}$

The finite element approximation is performed by choosing the functions $\widetilde{w}_{n}$ and $\widetilde{\theta}_{n}$ as a linear combination of given functions of $\xi$, usually polynomials. In a matrix form that reads:

$$
\left\{\begin{array}{c}
\widetilde{w}_{n}(\xi) \\
\widetilde{\theta}_{n}(\xi)
\end{array}\right\}=\underline{\underline{\mathbf{S}}}(\xi) \underline{\mathbf{W}}_{n},
$$

where $\underline{\mathbf{W}}_{n}$ is the vector of the element degrees of freedom, i.e. the coefficients of the linear combination, and $\underline{\underline{\mathbf{S}}}(\xi)$ is the two line matrix of the basis (or shape) functions. The functions of $x$ corresponding to the $\widetilde{w}_{n}$ and $\widetilde{\theta}_{n}$ through the relation (24) are denoted by $w^{d i s}(x)$ and $\theta^{d i s}(x)$ :

$$
\begin{aligned}
\widetilde{w}_{n}(\xi) & =w^{d i s}\left(\phi_{n}(\xi)\right), \\
\widetilde{\theta}_{n}(\xi) & =\theta^{d i s}\left(\phi_{n}(\xi)\right) .
\end{aligned}
$$

According to the Galerkin method, the algebraic equations satisfied by the degrees of freedom of the discretisation are found considering virtual fields that have the same form as the approximate unknowns, namely:

$$
\left\{\begin{array}{c}
\widetilde{w^{*}}{ }_{n}(\xi) \\
\widetilde{\theta_{n}^{*}}(\xi)
\end{array}\right\}=\underline{\underline{\mathbf{S}}}(\xi) \underline{\mathbf{W}}_{n}^{*} .
$$

The transfer of Eqs. 25 and 27 in Eqs. 22 and 23 straightforwardly yields:

$$
\begin{aligned}
a_{n}\left(\left(\widetilde{w}_{n}, \widetilde{\theta}_{n}\right),\left(\widetilde{w_{n}^{*}}, \widetilde{\theta_{n}^{*}}\right)\right) & =\underline{\mathbf{W}}_{n}^{* T} \underline{\mathbf{K}}_{n} \underline{\mathbf{W}}_{n}, \\
L_{n}\left(\widetilde{w_{n}^{*}}, \widetilde{\theta_{n}^{*}}\right) & =\underline{\mathbf{W}}_{n}^{* T} \underline{\mathbf{L}}_{n},
\end{aligned}
$$

where $\underline{\underline{\mathbf{K}}}^{n}$ is the dimensionless stiffness matrix of the element $E_{n}$ and $\underline{\mathbf{L}}_{n}$ is the element load vector.

Consequently, according to equations (20) and (21) the variational equation (9) becomes in the considered finite element 
approximation:

$$
\begin{aligned}
& \forall \underline{\mathbf{W}}^{*}, \sum_{n=1}^{N} \underline{\mathbf{W}}_{n}^{* T} \underline{\underline{\mathbf{K}}}_{n} \underline{\mathbf{W}}_{n}=\sum_{n=1}^{N} \underline{\mathbf{W}}_{n}^{* T} \underline{\mathbf{L}}_{n} \\
& +F_{0}^{F E} w_{0}^{*}+M_{0}^{F E} \theta_{0}^{*}+F_{1}^{F E} w_{1}^{*}+M_{1}^{F E} \theta_{1}^{*},
\end{aligned}
$$

where $\underline{\mathbf{W}}^{*}$ is the global column vector of the virtual degrees of freedom. The loadings at the two ends $x=0$ and $x=1$ of the beam are denoted $F_{0}^{F E}, M_{0}^{F E}, F_{1}^{F E}$ and $M_{1}^{F E}$, because, according to the considered problem, some of them can be unknowns and therefore are a priori only finite element approximations of the exact values given in section 4 .

\section{A new Timoshenko finite element beam}

The analytical solutions for a Timoshenko beam free from distributed loadings (or for $p=0$ and $m$ constant) have the form of a cubic function for the transverse displacements and a quadratic function for the rotations (see Eqs. 13a and 13b or Eqs. 14a and 14b). The main idea behind the development of the new FCQ Timoshenko finite element beam is to use shape functions that constitute a basis for degree three polynomials for the transverse displacements and for degree two polynomials for the rotations.

Remark: The Timoshenko finite element beam presented in [11] - with shape functions (cubic for the transverse displacements and quadratic for the rotations) depending on the material properties - should thus be a particular solution of the FCQ Timoshenko beam, (see Section 8 for the analytical proof).

More specifically:

- Four linearly independent polynomials are needed to form a basis for degree three polynomials. In a similar way, three linearly independent polynomials are needed to form a basis for degree two polynomials. That means that four degrees of freedom have to be used for the interpolation of $\widetilde{w}_{n}(\xi)$ and three for $\widetilde{\theta}_{n}(\xi)$.

- As the highest derivatives involved in the weak formulation (Eq. 9) are first derivatives for both $w$ and $\theta$, the continuity requirement for both discretised functions $w^{\text {dis }}$ and $\theta^{d i s}$ of Eqs. 26 is a $C^{0}$ continuity. To ensure the $C^{0}$ continuity of $w^{d i s}$ and $\theta^{d i s}$ two nodal degrees of freedom are sufficient for the $\widetilde{w}_{n}(\xi)$ and $\widetilde{\theta}_{n}(\xi)$ disretisations, one at $\xi=-1$ and one at $\xi=1$ of $S_{R}$.

- Consequently, the chosen discretisation of $\widetilde{w}_{n}(\xi)$ is based on two nodal and two internal degrees of freedom and that of $\widetilde{\theta}_{n}(\xi)$ is based on two nodal and one internal degrees of freedom.

- To define thus the discretisation of $\widetilde{w}_{n}(\xi)$ four independent polynomials of degree 3 at most are needed, one equal to 1 at $\xi=-1$ and to 0 at $\xi=1$, one equal to 0 at $\xi=-1$ and to 1 at $\xi=1$ and two equal to 0 at $\xi=-1$ and $\xi=1$. For the discretisation of $\widetilde{\theta}_{n}(\xi)$ three independent polynomials of degree 2 at most are needed, one equal to 1 at $\xi=-1$ and to 0 at $\xi=1$, one equal to 0 at $\xi=-1$ and to 1 at $\xi=1$ and one equal to 0 at $\xi=-1$ and $\xi=1$.

Obviously, one can find various basis functions that fulfil the above requirements. In the following, we draw inspiration from the conformal $C^{1}$ classical interpolation of the bending displacements of a Navier Bernoulli beam which reads $\left(w_{n}\right.$ and $\theta_{n}$ are the nodal degrees of freedom relative to node $n, w_{n+1}$ and $\theta_{n+1}$ the nodal degrees of freedom relative to node $n+1$ and $\Delta w_{n}^{1}$, $\Delta \theta_{n}, \Delta w_{n}^{2}$ the three internal degrees of freedom of the element, see also Eq. 39):

$$
\widetilde{w}_{n}(\xi)=w_{n} H_{R}^{1}(\xi)+\Delta w_{n}^{1} L_{R}^{1}(\xi)+\Delta w_{n}^{2} L_{R}^{2}(\xi)+w_{n+1} H_{R}^{2}(\xi)
$$

where $H_{R}^{1}, H_{R}^{2}, L_{R}^{1}, L_{R}^{2}$ are the four Hermite's polynomials on $S_{R}$ :

$$
\begin{aligned}
& H_{R}^{1}(\xi)=\frac{1}{4}(1-\xi)^{2}(2+\xi), \\
& H_{R}^{2}(\xi)=\frac{1}{4}(1+\xi)^{2}(2-\xi), \\
& L_{R}^{1}(\xi)=\frac{1}{4}(1-\xi)^{2}(1+\xi)=\frac{1}{4}\left(1-\xi^{2}\right)(1-\xi), \\
& L_{R}^{2}(\xi)=-\frac{1}{4}(1+\xi)^{2}(1-\xi)=-\frac{1}{4}\left(1-\xi^{2}\right)(1+\xi) .
\end{aligned}
$$

The derivation of Eq. 30 yields:

$$
\frac{d \widetilde{w}_{n}}{d \xi}=w_{n} \frac{d H_{R}^{1}}{d \xi}+\Delta w_{n}^{1} \frac{d L_{R}^{1}}{d \xi}+\Delta w_{n}^{2} \frac{d L_{R}^{2}}{d \xi}+w_{n+1} \frac{d H_{R}^{2}}{d \xi},
$$


with

$$
\begin{aligned}
\frac{d H_{R}^{1}}{d \xi} & =-\frac{3}{4}\left(1-\xi^{2}\right), \\
\frac{d H_{R}^{2}}{d \xi} & =\frac{3}{4}\left(1-\xi^{2}\right), \\
\frac{d L_{R}^{1}}{d \xi} & =-\frac{1}{4}(1-\xi)(1+3 \xi), \\
\frac{d L_{R}^{2}}{d \xi} & =-\frac{1}{4}(1+\xi)(1-3 \xi) .
\end{aligned}
$$

So, Eq. 32 reads:

$$
\frac{d \widetilde{w}_{n}}{d \xi}=\Delta w_{n}^{1} \frac{d L_{R}^{1}}{d \xi}+\Delta w_{n}^{2} \frac{d L_{R}^{2}}{d \xi}+\frac{3}{4}\left(w_{n+1}-w_{n}\right)\left(1-\xi^{2}\right) .
$$

In a similar way, we discretise the rotation $\widetilde{\theta}_{n}$ as follows:

$$
\widetilde{\theta}_{n}(\xi)=\theta_{n} \frac{d L_{R}^{1}}{d \xi}(\xi)+\Delta \theta_{n} M_{R}(\xi)+\theta_{n+1} \frac{d L_{R}^{2}}{d \xi}(\xi)
$$

where $M_{R}(\xi)$ is:

$$
M_{R}(\xi)=1-\xi^{2}
$$

\section{Remarks:}

- Different choices exist in the literature for the interpolation polynomials of $\widetilde{w}_{n}(\xi)$ and $\widetilde{\theta}_{n}(\xi)$, (see for example [19], [20], [23], [24], [25]).

- The interest of Eqs. 30 and 36 is that they obviously include the classical $C^{1}$ conforming interpolation used for Navier Bernoulli beams, and thus insure that the FCQ Timoshenko beam is free of shear locking.

- Clearly, the interpolations of Eqs. 30 and 36 span the spaces of degree 3 and degree 2 polynomials in $\xi$ respectively. Therefore, and due to the affine character of the change of variables given by Eqs. 17 and 24, the corresponding functions $w_{n}^{d i s}(x)$ and $\theta_{n}^{d i s}(x)$ in the element $E_{n}$ span the spaces of respectively degree 3 and degree 2 polynomials in $x$.

- Consequently, the exact solution $\left(w^{\text {exa }}, \theta^{\text {exa }}\right)$ given by Eqs. 13 for a Timoshenko beam free from distributed loadings or for $p=0$ and $m$ constant is in the space spanned by the discretised functions $w_{n}^{d i s}(x)$ and $\theta_{n}^{d i s}(x)$ with only one element. In other words, a unique FCQ Timoshenko element provides the exact solution of the bending problem for a Timoshenko beam free from distributed loadings or for $p=0$ and $m$ constant whatever the boundary conditions are.

In a matrix format, the two line matrix of the shape functions $\underline{\underline{\mathbf{S}}}(\xi)$ of Eq. 25 corresponding to the interpolation of Eqs. 30 and 36 reads:

$$
\underline{\underline{\mathbf{S}}}(\xi)=\left[\begin{array}{ccccccc}
H_{R}^{1}(\xi) & 0 & L_{R}^{1}(\xi) & 0 & L_{R}^{2}(\xi) & H_{R}^{2}(\xi) & 0 \\
0 & \frac{d L_{R}^{1}(\xi)}{d \xi} & 0 & M_{R}(\xi) & 0 & 0 & \frac{d L_{R}^{2}(\xi)}{d \xi}
\end{array}\right]
$$

and the column vector of the element degrees of freedom $\underline{\mathbf{W}}_{n}$ is:

$$
\underline{\mathbf{W}}_{n}=\left\{\begin{array}{c}
w_{n} \\
\theta_{n} \\
\Delta w_{n}^{1} \\
\Delta \theta_{n} \\
\Delta w_{n}^{2} \\
w_{n+1} \\
\theta_{n+1}
\end{array}\right\},
$$

where $w_{n}$ and $\theta_{n}$ are the nodal degrees of freedom relative to node $n, w_{n+1}$ and $\theta_{n+1}$ the nodal degrees of freedom relative to node $n+1$ and $\Delta w_{n}^{1}, \Delta \theta_{n}, \Delta w_{n}^{2}$ the three internal degrees of freedom of the element.

Following the general procedure described in Section 5, the $7 \times 7$ element stiffness matrix takes the form (calculations are made using the GNU General Public License (GPL) software Maxima [16]):

$\underline{\underline{\mathbf{K}}}_{n}=\frac{1}{30 e l_{n}}$

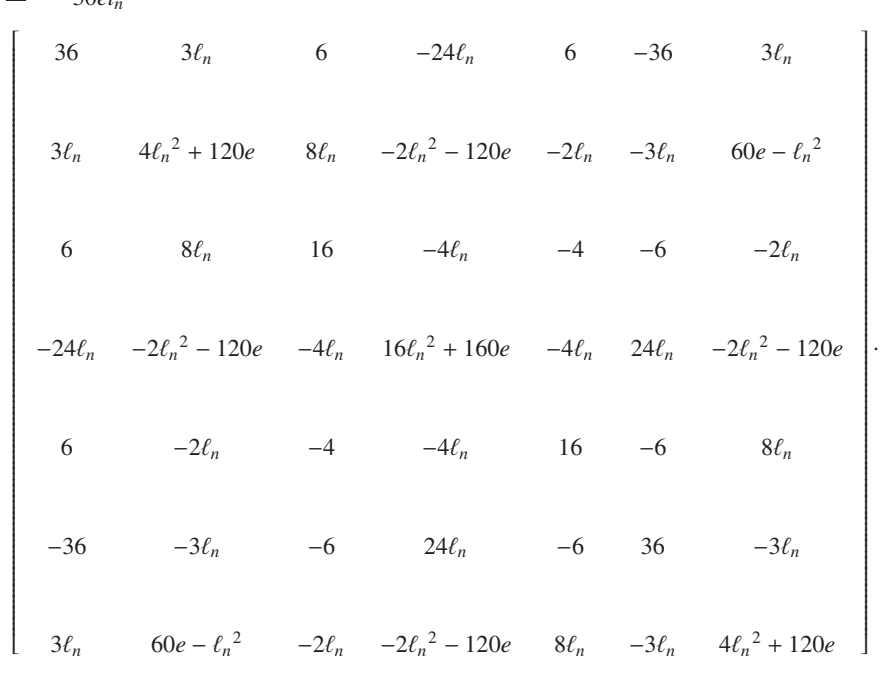




\section{Static condensation}

To implement the new Timoshenko finite element beam to a general purpose finite element code we have to solve for the internal degrees of freedom. There are two ways to do this:

- Either the internal degrees of freedom are treated as typical (external) degrees of freedom, meaning that they are sent to the global solver of the finite element code (see Section 10.3)

- Either they are calculated locally in the element using the static condensation method.

Static condensation is the process of reducing the number of degrees of freedom by the elimination of some unknowns (see [6] for instance). It is particularly interesting when it's applied to internal degrees of freedom of an element because it is performed at the element level (element's stiffness matrix and load vector) and not at the global level.

To make that clearer, let's separate the internal degrees of freedom $\Delta w_{n}^{1}, \Delta \theta_{n}, \Delta w_{n}^{1}$ from the nodal ones $w_{n}, \theta_{n}, w_{n+1}, \theta_{n+1}$ by writing the real as well the virtual column vectors $\underline{\mathbf{W}}_{n}$ and $\underline{\mathbf{W}}_{n}^{*}$ in form of block vectors:

$$
\underline{\mathbf{W}}_{n}=\left\{\begin{array}{l}
\underline{\mathbf{W}}_{n}^{1} \\
\underline{\mathbf{W}}_{n}^{2} \\
\underline{\mathbf{W}}_{n}^{3}
\end{array}\right\}, \underline{\mathbf{W}}_{n}^{*}=\left\{\begin{array}{l}
\underline{\mathbf{W}}_{n}^{1 *} \\
\underline{\mathbf{W}}_{n}^{2 *} \\
\underline{\mathbf{W}}_{n}^{3 *}
\end{array}\right\},
$$

where

$$
\underline{\mathbf{W}}_{n}^{1}=\left\{\begin{array}{c}
w_{n} \\
\theta_{n}
\end{array}\right\}, \underline{\mathbf{W}}_{n}^{2}=\left\{\begin{array}{c}
\Delta w_{n}^{1} \\
\Delta \theta_{n} \\
\Delta w_{n}^{2}
\end{array}\right\}, \underline{\mathbf{W}}_{n}^{3}=\left\{\begin{array}{c}
w_{n+1} \\
\theta_{n+1}
\end{array}\right\},
$$

and $\underline{\mathbf{W}}_{n}^{1 *}, \underline{\mathbf{W}}_{n}^{2 *}, \underline{\mathbf{W}}_{n}^{3 *}$ defined accordingly. The element nodal degrees of freedom are gathered in the 4 lines column vector $\underline{\mathbf{W}}_{n}^{B}$ :

$$
\underline{\mathbf{W}}_{n}^{B}=\left\{\begin{array}{l}
w_{n} \\
\theta_{n} \\
w_{n+1} \\
\theta_{n+1}
\end{array}\right\}=\left\{\begin{array}{l}
\underline{\mathbf{W}}_{n}^{1} \\
\underline{\mathbf{W}}_{n}^{3}
\end{array}\right\} .
$$

The element load vector $\underline{\mathbf{L}}_{n}$ and stiffness matrix $\underline{\mathbf{K}}_{n}$ are written accordingly:

$$
\underline{\mathbf{L}}_{n}=\left\{\begin{array}{l}
\underline{\mathbf{L}}_{n}^{1} \\
\underline{\mathbf{L}}_{n}^{2} \\
\underline{\mathbf{L}}_{n}^{3}
\end{array}\right\}, \underline{\underline{\mathbf{K}}}_{n}=\left[\begin{array}{lll}
\underline{\underline{\mathbf{K}}}_{n}^{11} & \underline{\underline{\mathbf{K}}}_{n}^{12} & \underline{\underline{\mathbf{K}}}_{n}^{13} \\
\underline{\underline{\mathbf{K}}}_{n}^{21} & \underline{\mathbf{K}}_{n}^{22} & \underline{\underline{\mathbf{K}}}_{n}^{23} \\
\underline{\underline{\mathbf{K}}}_{n}^{31} & \underline{\mathbf{K}}_{n}^{32} & \underline{\underline{\mathbf{K}}}_{n}^{33}
\end{array}\right],
$$

where $\underline{\mathbf{L}}_{n}^{1}$ and $\underline{\mathbf{L}}_{n}^{3}$ are $2 \times 1$ vectors; $\underline{\mathbf{L}}_{n}^{2}$ is a $3 \times 1$ vector; $\underline{\mathbf{K}}_{n}^{11}$, $\underline{\underline{\mathbf{K}}}_{n}^{13}, \underline{\underline{\mathbf{K}}}_{n}^{31}$ and $\underline{\underline{\mathbf{K}}}_{n}^{33}$ are $2 \times 2$ matrices; $\underline{\underline{\mathbf{K}}}_{n}^{12}$ and $\underline{\underline{\mathbf{K}}}_{n}^{32}$ are $2 \times 3$ matrices; $\underline{\underline{\mathbf{K}}}_{n}^{21}$ and $\underline{\underline{\mathbf{K}}}_{n}^{23}$ are $3 \times 2$ matrices and $\underline{\mathbf{K}}_{n}^{22}$ is a $3 \times 3$ matrix.

Remark: As the element stiffness matrix is symmetric, the blocks satisfy the relation:

$$
\underline{\underline{\mathbf{K}}}_{n}^{i j T}=\underline{\underline{\mathbf{K}}}_{n}^{j i}, i, j=1,2,3 \text {. }
$$

Following the previous notations, the element bilinear and linear forms of Eqs. 28a, 28b and 29 read:

$\underline{\mathbf{W}}_{n}^{* T} \underline{\underline{\mathbf{K}}}_{n} \underline{\mathbf{W}}_{n}=$

$$
\begin{aligned}
& \left\{\begin{array}{ll}
\underline{\mathbf{W}}_{n}^{1 * T} & \underline{\mathbf{W}}_{n}^{3 * T}
\end{array}\right\}\left(\left[\begin{array}{ll}
\underline{\underline{\mathbf{K}}}_{n}^{11} & \underline{\underline{\mathbf{K}}}_{n}^{13} \\
\underline{\underline{\mathbf{K}}}_{n}^{31} & \underline{\underline{\mathbf{K}}}_{n}^{33}
\end{array}\right]\left\{\begin{array}{l}
\underline{\mathbf{W}}_{n}^{1} \\
\underline{\mathbf{W}}_{n}^{3}
\end{array}\right\}+\left[\begin{array}{l}
\underline{\underline{\mathbf{K}}}_{n}^{12} \\
\underline{\underline{\mathbf{K}}}_{n}^{32}
\end{array}\right] \underline{\mathbf{W}}_{n}^{2}\right) \\
& +\underline{\mathbf{W}}_{n}^{2 * T}\left(\underline{\underline{\mathbf{K}}}_{n}^{22} \underline{\mathbf{W}}_{n}^{2}+\left[\begin{array}{ll}
\underline{\mathbf{K}}_{n}^{21} & \underline{\mathbf{K}}_{n}^{23}
\end{array}\right]\left\{\begin{array}{l}
\underline{\mathbf{W}}_{n}^{1} \\
\underline{\mathbf{W}}_{n}^{3}
\end{array}\right\}\right), \\
& \underline{\mathbf{W}}_{n}^{* T} \underline{\mathbf{L}}_{n}=\left\{\underline{\mathbf{W}}_{n}^{1 * T} \quad \underline{\mathbf{W}}_{n}^{3 * T}\right\}\left\{\begin{array}{c}
\underline{\mathbf{L}}_{n}^{1} \\
\underline{\mathbf{L}}_{n}^{3}
\end{array}\right\}+\underline{\mathbf{W}}_{n}^{2 * T} \underline{\mathbf{L}}_{n}^{2} .
\end{aligned}
$$

Using the notations 43, that reads too:

$$
\begin{aligned}
& \underline{\mathbf{W}}_{n}^{* T} \underline{\mathbf{K}}_{n} \underline{\mathbf{W}}_{n}= \\
& \underline{\mathbf{W}}_{n}^{B \star T}\left(\left[\begin{array}{ll}
\underline{\underline{\mathbf{K}}}_{n}^{11} & \underline{\mathbf{K}}_{n}^{13} \\
\underline{\underline{\mathbf{K}}}_{n}^{31} & \underline{\mathbf{K}}_{n}^{33}
\end{array}\right] \underline{\mathbf{W}}_{n}^{B}+\left[\begin{array}{l}
\underline{\underline{\mathbf{K}}}_{n}^{12} \\
\underline{\mathbf{K}}_{n}^{32}
\end{array}\right] \underline{\mathbf{W}}_{n}^{2}\right) \\
& +\underline{\mathbf{W}}_{n}^{2 * T}\left(\underline{\underline{\mathbf{K}}}_{n}^{22} \underline{\mathbf{W}}_{n}^{2}+\left[\begin{array}{ll}
\underline{\mathbf{K}}_{n}^{21} & \underline{\mathbf{K}}_{n}^{23}
\end{array}\right] \underline{\mathbf{W}}_{n}^{B}\right), \\
& \underline{\mathbf{W}}_{n}^{* T} \underline{\mathbf{L}}_{n}=\underline{\mathbf{W}}_{n}^{B \star T}\left\{\begin{array}{l}
\underline{\mathbf{L}}_{n}^{1} \\
\underline{\mathbf{L}}_{n}^{3}
\end{array}\right\}+\underline{\mathbf{W}}_{n}^{2 * T} \underline{\mathbf{L}}_{n}^{2} .
\end{aligned}
$$

where $\underline{\mathbf{W}}_{n}^{B \star T}$ is defined from $\underline{\mathbf{W}}_{n}^{1 \star}$ and $\underline{\mathbf{W}}_{n}^{3 \star}$ accordingly to Eq. 43.

In the following, we are going to calculate the expressions for the reduced element stiffness matrix and load vector. Eq. 29 
is valid for any virtual global column vector $\underline{\mathbf{W}}^{*}$. Considering the virtual degrees of freedom at the boundaries of a specific element $E_{n}$ equal to zero and all the virtual degrees of freedom of all the other elements $E_{m}(1 \leq m \leq N, m \neq n)$ equal to zero, we get $\underline{\mathbf{W}}_{n}^{* T}=\left\{\begin{array}{ccccc}0 & 0 & \underline{\mathbf{W}}_{n}^{2 * T} & 0 & 0\end{array}\right\}$, that is $\underline{\mathbf{W}}_{n}^{B \star T}=\underline{0}$, and $\underline{\mathbf{W}}_{m}^{*}=\underline{\mathbf{0}}$ where $\underline{\mathbf{0}}$ is the zero vector. Eqs. 29, 48 and 49 yield:

$$
\forall \underline{\mathbf{W}}_{n}^{2 *}, \underline{\mathbf{W}}_{n}^{2 * T}\left(\underline{\underline{\mathbf{K}}}_{n}^{22} \underline{\mathbf{W}}_{n}^{2}+\left[\underline{\mathbf{K}}_{n}^{21} \quad \underline{\mathbf{K}}_{n}^{23}\right] \quad \underline{\mathbf{W}}_{n}^{B}\right)=\underline{\mathbf{W}}_{n}^{2 * T} \underline{\mathbf{L}}_{n}^{2}
$$

As $\underline{\mathbf{W}}_{n}^{2 *}$ is any $3 \times 1$ vector, the previous equation is equivalent to the linear system:

$$
\underline{\underline{\mathbf{K}}}_{n}^{22} \underline{\mathbf{W}}_{n}^{2}+\left[\underline{\underline{\mathbf{K}}}_{n}^{21} \quad \underline{\mathbf{K}}_{n}^{23}\right] \underline{\mathbf{W}}_{n}^{B}=\underline{\mathbf{L}}_{n}^{2}
$$

The purpose of the static condensation is to eliminate $\underline{\mathbf{W}}_{n}^{2}$ by expressing it in terms of the other degrees of freedom and applied forces, that its say, considering Eq. 51, in terms of $\underline{\mathbf{W}}_{n}^{B}$ and $\underline{\mathbf{L}}_{n}^{2}$. Let's pose:

$$
\begin{aligned}
& \underline{\mathbf{W}}_{n}^{2 a}=-\left(\underline{\underline{\mathbf{K}}}_{n}^{22}\right)^{-1}\left[\begin{array}{ll}
\underline{\mathbf{K}}_{n}^{21} & \underline{\mathbf{K}}_{n}^{23}
\end{array}\right] \underline{\mathbf{W}}_{n}^{B}, \\
& \underline{\mathbf{W}}_{n}^{2 b}=\left(\underline{\underline{\mathbf{K}}}_{n}^{22}\right)^{-1} \underline{\mathbf{L}}_{n}^{2} .
\end{aligned}
$$

Thanks to the linearity of Eq. 51 it can be seen that $\underline{\mathbf{W}}_{n}^{2}$ reads:

$$
\underline{\mathbf{W}}_{n}^{2}=\underline{\mathbf{W}}_{n}^{2 a}+\underline{\mathbf{W}}_{n}^{2 b}
$$

Following the static condensation the discretised fields of Eqs. 30 and 36 read:

$$
\left\{\begin{array}{c}
\widetilde{w}_{n}(\xi) \\
\widetilde{\theta}_{n}(\xi)
\end{array}\right\}=\left\{\begin{array}{c}
\widetilde{w}_{n}^{a}(\xi) \\
\widetilde{\theta}_{n}^{a}(\xi)
\end{array}\right\}+\left\{\begin{array}{c}
\widetilde{w}_{n}^{b}(\xi) \\
\widetilde{\theta}_{n}^{b}(\xi)
\end{array}\right\},
$$

with

$$
\begin{gathered}
\left\{\begin{array}{c}
\widetilde{w}_{n}^{a}(\xi) \\
\widetilde{\theta}_{n}^{a}(\xi)
\end{array}\right\}=\underline{\underline{\mathbf{S}}}(\xi)\left\{\begin{array}{c}
\underline{\mathbf{W}}_{n}^{1} \\
\underline{\mathbf{W}}_{n}^{2 a} \\
\underline{\mathbf{W}}_{n}^{3}
\end{array}\right\}, \\
\left\{\begin{array}{c}
\underline{w}_{n}^{b}(\xi) \\
\widetilde{\theta}_{n}^{b}(\xi)
\end{array}\right\}=\underline{\underline{\mathbf{S}}}(\xi)\left\{\begin{array}{c}
\underline{\mathbf{W}}_{n}^{2 b} \\
0 \\
0 \\
0
\end{array}\right\} .
\end{gathered}
$$

Introducing Eq. 54 in Eq. 46 we get:

$$
\begin{aligned}
& \underline{\mathbf{W}}_{n}^{* T} \underline{\underline{\mathbf{K}}}_{n} \underline{\mathbf{W}}_{n}= \\
& \underline{\mathbf{W}}_{n}^{B \star T} \underline{\underline{\mathbf{K}}}_{n}^{r e d} \underline{\mathbf{W}}_{n}^{B} \\
& +\underline{\mathbf{W}}_{n}^{B \star T}\left[\begin{array}{l}
\underline{\underline{\mathbf{K}}}_{n}^{12} \\
\underline{\underline{\mathbf{K}}}_{n}^{32}
\end{array}\right]\left(\underline{\underline{\mathbf{K}}}_{n}^{22}\right)^{-1} \underline{\mathbf{L}}_{n}^{2}+\underline{\mathbf{W}}_{n}^{2 * T} \underline{\mathbf{L}}_{n}^{2},
\end{aligned}
$$

where the reduced $4 \times 4$ element stiffness marix $\underline{\underline{\mathbf{K}}}_{n}^{\text {red }}$ reads:

$$
\underline{\underline{\mathbf{K}}}_{n}^{r e d}=\left[\begin{array}{ll}
\underline{\underline{\mathbf{K}}}_{n}^{11} & \underline{\underline{\mathbf{K}}}_{n}^{13} \\
\underline{\underline{\mathbf{K}}}_{n}^{31} & \underline{\underline{\mathbf{K}}}_{n}^{33}
\end{array}\right]-\left[\begin{array}{l}
\underline{\underline{\mathbf{K}}}_{n}^{12} \\
\underline{\underline{\mathbf{K}}}_{n}^{32}
\end{array}\right]\left(\underline{\underline{\mathbf{K}}}_{n}^{22}\right)^{-1}\left[\begin{array}{ll}
\underline{\mathbf{K}}_{n}^{21} & \underline{\mathbf{K}}_{n}^{23}
\end{array}\right] .
$$

Eqs. 58 and 29 represent the contribution of the element $E_{n}$ to the left-hand side or respectively the right-hand side of the discretised weak formulation (29).

The term $\underline{\mathbf{W}}_{n}^{2 * T} \underline{\mathbf{L}}_{n}^{2}$ vanishes when the two expressions (Eqs. 49 and 58) are transferred into Eq. 29, so the contribution of the element $E_{n}$ to the weak formulation of (29) is thus the bilinear form:

$$
\underline{\mathbf{W}}_{n}^{B \star T} \underline{\underline{\mathbf{K}}}_{n}^{r e d} \underline{\mathbf{W}}_{n}^{B}
$$

for the left-hand side and the linear form:

$$
\underline{\mathbf{W}}_{n}^{B \star T} \underline{\mathbf{L}}_{n}^{\text {red }}
$$

for the right hand side where the reduced $4 \times 1$ element load vector $\underline{\mathbf{L}}_{n}^{\text {red }}$ reads:

$$
\underline{\mathbf{L}}_{n}^{r e d}=\left\{\begin{array}{l}
\underline{\mathbf{L}}_{n}^{1} \\
\underline{\mathbf{L}}_{n}^{3}
\end{array}\right\}-\left[\begin{array}{l}
\underline{\underline{\mathbf{K}}}_{n}^{12} \\
\underline{\underline{\mathbf{K}}}_{n}^{32}
\end{array}\right]\left(\underline{\underline{\mathbf{K}}}_{n}^{22}\right)^{-1} \underline{\mathbf{L}}_{n}^{2}
$$

The previous developments show that the static condensation can be performed element by element, previous to the assembly of the global stiffness matrix and load vector.

For the specific case of the FCQ Timoshenko beam element we successively get:

$$
\left(\underline{\underline{\mathbf{K}}}_{n}^{22}\right)^{-1}=\frac{3 e \ell_{n}}{4\left(\ell_{n}^{2}+12 e\right)}\left[\begin{array}{ccc}
3 \ell_{n}^{2}+32 e & \ell_{n} & \ell_{n}^{2}+8 e \\
\ell_{n} & 3 & \ell_{n} \\
\ell_{n}^{2}+8 e & \ell_{n} & 3 \ell_{n}^{2}+32 e
\end{array}\right] \text {, }
$$


then:

$$
\begin{aligned}
& \underline{\mathbf{W}}_{n}^{2 a}=\frac{1}{2\left(\ell_{n}^{2}+12 e\right)} \\
& {\left[\begin{array}{cccc}
-12 e & -\left(\ell_{n}^{3}+6 e \ell_{n}\right) & 12 e & 6 e \ell_{n} \\
3 \ell_{n} & 18 e & -3 \ell_{n} & 18 e \\
-12 e & 6 e \ell_{n} & 12 e & -\left(\ell_{n}^{3}+6 e \ell_{n}\right)
\end{array}\right] \underline{\mathbf{W}}_{n}^{B},}
\end{aligned}
$$

where $\underline{\mathbf{W}}_{n}^{B}$ is defined in Eq. 43 and:

$$
\begin{aligned}
& \underline{\underline{\mathbf{K}}}_{n}^{\text {red }}= \\
& \frac{2}{\ell_{n}\left(\ell_{n}^{2}+12 e\right)}\left[\begin{array}{cccc}
6 & -3 \ell_{n} & -6 & -3 \ell_{n} \\
-3 \ell_{n} & 2 \ell_{n}^{2}+6 e & 3 \ell_{n} & \ell_{n}^{2}-6 e \\
-6 & 3 \ell_{n} & 6 & 3 \ell_{n} \\
-3 \ell_{n} & \ell_{n}^{2}-6 e & 3 \ell_{n} & 2 \ell_{n}^{2}+6 e
\end{array}\right] .
\end{aligned}
$$

As $\underline{\mathbf{W}}^{2 a}$ linearly depends on $\underline{\mathbf{W}}^{B}$, the interpolation of Eq. 56 can be written:

$$
\left\{\begin{array}{c}
\widetilde{w}_{n}^{a}(\xi) \\
\widetilde{\theta}_{n}^{a}(\xi)
\end{array}\right\}=\underline{\underline{\mathbf{S}}}^{r e d}(\xi) \underline{\mathbf{W}}_{n}^{B} .
$$

When only one element is used $\left(\ell_{n}=1\right)$, the reduced stiffness matrix $\underline{\mathbf{K}}_{1}^{\text {red }}$ of Eq. 64 coincides with the analytical stiffness matrix of a Timoshenko beam free of distributed loadings (see Eq. 16). The bending problem of a Timoshenko beam loaded only at its ends is therefore exactly solved with only one FCQ element.

Indeed, in that case the variational equation (Eq. 29) reads:

$$
\begin{aligned}
& \forall \underline{\mathbf{W}}_{1}^{B *}=\left(w_{0}^{*}, \theta_{0}^{*}, w_{1}^{*}, \theta_{1}^{*}\right)^{T}, \\
& \underline{\mathbf{W}}_{1}^{B * T} \underline{\mathbf{K}}_{1}^{\text {red }} \underline{\mathbf{W}}_{1}^{B}=F_{0}^{F E} w_{0}^{*}+M_{0}^{F E} \theta_{0}^{*}+F_{1}^{F E} w_{1}^{*}+M_{1}^{F E} \theta_{1}^{*},
\end{aligned}
$$

with $\underline{\mathbf{W}}_{1}^{B}=\left(w_{0}, \theta_{0}, w_{1}, \theta_{1}\right)^{T}$, which obviously yields Eq. 15 . This result is consistent with the remark of section 6 page 6 , where it is mentioned that for the case of a Timoshenko beam free of distributed loadings one element gives the exact solution of the beam bending. In line with that remark, the pair $\left(w_{1}^{a}(x), \theta_{1}^{a}(x)\right)$ corresponding to the pair $\left(\widetilde{w}_{1}^{a}(\xi), \widetilde{\theta}_{1}^{a}(\xi)\right)$ of Eq. 65 through the change of variables of Eq. 24, exactly satisfies the balance and constitutive Eqs. 3 and 4.

\section{Comparison with the formulation presented in [11]}

The purpose of this section is to prove that the element introduced by Friedman and Kosmatka can be derived from the present formulation. In [11], the two authors have introduced a two node Timoshenko finite element beam using cubic and quadratic polynomials for the transverse displacements and rotations respectively. The polynomials are made interdependent by requiring them to satisfy the two homogeneous differential equations associated with Timoshneko's beam theory. The resulting stiffness matrix is exactly integrated and the element is free of shear locking. The main limitation of this element is that the proposed interpolation functions depend on the materials properties: this is at odds with the finite element method and limits the domain of application of the element to linear elastic problems (although some satisfactory results can be found in the literature for non linear materials by keeping the original interpolation functions unchanged, see for example [14] and [17]). If however the values of the original interpolation functions are updated, numerical convergence problems soon appear.

The interpolation used in [11] takes the following form:

$$
\left\{\begin{array}{c}
\widetilde{w}_{n}^{F K}(\xi) \\
\widetilde{\theta}_{n}^{F K}(\xi)
\end{array}\right\}=\left[\begin{array}{cccc}
N_{n}^{1}(\xi) & N_{n}^{2}(\xi) & N_{n}^{3}(\xi) & N_{n}^{4}(\xi) \\
N_{n}^{5}(\xi) & N_{n}^{6}(\xi) & N_{n}^{7}(\xi) & N_{n}^{8}(\xi)
\end{array}\right] \underline{\mathbf{W}}_{n}^{B},
$$

where $\underline{\mathbf{W}}_{n}^{B}$ is defined by Eq. 43. Details on the interpolation functions $N_{n}^{i}, i=1,8$ that depend on the material's properties can be found in [11].

As defined by Friedman and Kosmatka, the interpolation of Eq. 67 is such that it solves exactly the bending equations of a Timoshenko beam free of distributed loading whatever the $\underline{\mathbf{W}}_{n}^{B}$ is (as the interpolation functions satisfy the two homogeneous differential equations associated with Timoshneko's beam theory). In accordance with the comments presented at the end of the previous section, the interpolation of Eq. 67 - up to the change of variables (Eq. 24) - is identical to the interpolation of Eq. 65, which means that $\widetilde{w}_{n}^{F K}(\xi)=\widetilde{w}_{n}^{a}(\xi)$ and $\widetilde{\theta}_{n}^{F K}=\widetilde{\theta}_{n}^{a}$. Therefore, in order to prove that the element presented in [11] can be derived from the new FCQ element formulation, it is suffi- 
cient to show that the two discretised problems are the same, in other words that the element stiffness matrices and element load vectors are identical.

According to Eq. 28a, the element stiffness matrix $\underline{\underline{\mathbf{K}}}_{n}^{F K}$ introduced by Friedman and Kosmatka in [11] (corresponding to the interpolation of Eq. 67 which is identical to the interpolation interpolation of Eq. 65), is such that:

$$
\underline{\mathbf{W}}_{n}^{B * T} \underline{\underline{\mathbf{K}}}_{n}^{F K} \underline{\mathbf{W}}_{n}^{B}=a_{n}\left(\left(\widetilde{w}_{n}^{a}, \widetilde{\theta}_{n}^{a}\right),\left(\widetilde{w}_{n}^{a *}, \widetilde{\theta}_{n}^{a *}\right)\right),
$$

where the virtual fields $\widetilde{w}_{n}^{a *}$ and $\widetilde{\theta}_{n}^{a *}$ depend on $\underline{\mathbf{W}}_{n}^{B *}$ in the same way as the fields $\widetilde{w}_{n}^{a}$ and $\widetilde{\theta}_{n}^{a}$ depend on $\underline{\mathbf{W}}_{n}^{B}$ through Eqs. 56 and 52 .

The discretisations (Eq. 56) of $\widetilde{w}_{n}^{a}$ and of $\widetilde{\theta}_{n}^{a}$ use the same matrix $\underline{\underline{S}}(\xi)$ of basis functions as those of $\widetilde{w}_{n}$ and of $\widetilde{\theta}_{n}$ (Eq. 25). Therefore, according to Eq. 28a we have:

$$
\begin{aligned}
& \underline{\mathbf{W}}_{n}^{B * T} \underline{\underline{K}}_{n}^{F K} \underline{\mathbf{W}}_{n}^{B}= \\
& \left\{\begin{array}{lll}
\underline{\mathbf{W}}_{n}^{1 * T} & \underline{\mathbf{W}}_{n}^{2 a * T} & \underline{\mathbf{W}}_{n}^{3 * T}
\end{array}\right\} \underline{\mathbf{K}}_{n}\left\{\begin{array}{l}
\underline{\mathbf{W}}_{n}^{1} \\
\underline{\mathbf{W}}_{n}^{2 a} \\
\underline{\mathbf{W}}_{n}^{3}
\end{array}\right\}
\end{aligned}
$$

The expansion of Eq. 69 reads as Eq. 48 but with $\underline{\mathbf{W}}_{n}^{2 a}$ replacing $\underline{\mathbf{W}}_{n}^{2}$ and according to Eqs. 52, 59, it comes:

$$
\underline{\mathbf{W}}_{n}^{B * T} \underline{\underline{\mathbf{K}}}_{n}^{F K} \underline{\mathbf{W}}_{n}^{B}=\underline{\mathbf{W}}_{n}^{B \star T} \underline{\underline{\mathbf{K}}}_{n}^{r e d} \underline{\mathbf{W}}_{n}^{2 a}
$$

which proves that:

$$
\underline{\underline{\mathbf{K}}}_{n}^{F K}=\underline{\underline{\mathbf{K}}}_{n}^{\text {red }}
$$

where $\underline{\underline{\mathbf{K}}}_{n}^{\text {red }}$ is given by Eq. 64 .

According to Eqs. 28b, 49 and 56 a similar reasoning applied to the linear form $\underline{\mathbf{W}}_{n}^{B * T} \underline{\mathbf{L}}_{n}^{F K}$ yields:

$$
\begin{aligned}
\underline{\mathbf{W}}_{n}^{B * T} \underline{\mathbf{L}}_{n}^{F K} & =L_{n}\left(\widetilde{w}_{n}^{a}, \widetilde{\theta}_{n}^{a}\right) \\
& =\underline{\mathbf{W}}_{n}^{B * T}\left\{\begin{array}{l}
\underline{\mathbf{L}}_{n}^{1} \\
\underline{\mathbf{L}}_{n}^{3}
\end{array}\right\}+\underline{\mathbf{W}}_{n}^{2 a * T} \underline{\mathbf{L}}_{n}^{2} .
\end{aligned}
$$

$\underline{\mathbf{W}}_{n}^{2 a *}$ depends on $\underline{\mathbf{W}}_{n}^{B *}$ in the same way as $\underline{\mathbf{W}}_{n}^{2 a}$ depends on $\underline{\mathbf{W}}_{n}^{B}$. Using Eq. 52 and the symmetry (Eq. 45) of the matrix $\underline{\underline{\mathbf{K}}}_{n}$, we have:

$$
\underline{\mathbf{W}}_{n}^{2 a * T}=-\underline{\mathbf{W}}_{n}^{B * T}\left[\begin{array}{l}
\underline{\underline{\mathbf{K}}}_{n}^{21} \\
\underline{\underline{\mathbf{K}}}_{n}^{23}
\end{array}\right]\left(\underline{\underline{\mathbf{K}}}_{n}^{22}\right)^{-1}
$$

then, comparing Eq. 71 with Eq. 62, we finally obtain:

$$
\underline{\mathbf{L}}_{n}^{F K}=\underline{\mathbf{L}}_{n}^{r e d}
$$

Eqs. 70 and 73 prove that for the same number of elements the FCQ and the Friedman and Kosmatka Timoshenko beam elements give identical results for the degrees of freedom $w_{n}$ and $\theta_{n}$. Nevertheless, the FCQ beam adopts the discretisation provided by Eq. 55, whereas the Friedman and Kosmatka element by Eq. 56. According to Eqs. 30, 57, the difference in $\widetilde{w}_{n}^{b}$ and $\widetilde{\theta}_{n}^{b}(\xi)$ which reads:

$$
\left\{\begin{array}{c}
\widetilde{w}_{n}^{b}(\xi) \\
\widetilde{\theta}_{n}^{b}(\xi)
\end{array}\right\}=\left\{\begin{array}{ccc}
L_{R}^{1}(\xi) & 0 & L_{R}^{1}(\xi) \\
0 & M_{R}(\xi) & 0
\end{array}\right\} \underline{\mathbf{W}}_{n}^{2 b},
$$

influences the approximation of the shear force $T$ and of the bending moment $M$. This can be crucial when an anelasticity threshold is adopted based on the value of $T$ or $M$.

\section{Analytical proof for complex distributed loadings}

In [11], the authors numerically verified that one element with the interpolation functions of Eq. 67 is able to predict the exact tip displacement of a cantilever Timoshenko beam subjected to either an applied transverse tip load, a uniform load, or a linear varying distributed load. The purpose of this section is to prove that the result holds true for any complex distributed loads, moments included, and any suitable boundary conditions.

Let's consider a pair $(w, \theta)$ of functions satisfying the Eqs. 3 and 4 for given distributed loadings $p$ and $m$ and boundary conditions (Eq. 6) (as pointed out in section 2.2, the boundary conditions satisfy the compatibility conditions (Eq. 15)). Therefore, the pair $(w, \theta)$ satisfies the variational equation (Eq. 9) of section 3 that reads:

$$
\begin{aligned}
& \forall\left(w^{*}, \theta^{*}\right), a\left((w, \theta),\left(w^{*}, \theta^{*}\right)\right)=L\left(w^{*}, \theta^{*}\right) \\
& +F_{0} w_{0}^{*}+M_{0} \theta_{0}^{*}+F_{1} w_{1}^{*}+M_{1} \theta_{1}^{*},
\end{aligned}
$$

where $a\left((w, \theta),\left(w^{*}, \theta^{*}\right)\right)$ and $L\left(w^{*}, \theta^{*}\right)$ are given by Eqs. 10 and 11. 
Let's now consider the finite element approximation of the pair $(w, \theta)$ with one single element and the interpolation given by Eq. 65 (or equivalently of Eq. 67). To that purpose let $V^{1}$ denotes the set of pair fields $(w, \theta)$ of the form of Eq. 65 for $n=1$, that is to say the set of pairs $(w, \theta)$ verifying Eqs. 3 and 4 for $p=0$ and $m=0$.

In that one finite element simulation, the approximation $\left(w^{F E 1}, \theta^{F E 1}\right)$ of the pair $(w, \theta)$ verifies the following variational form obtained from Eq. 74 by considering $\left(w^{*}, \theta^{*}\right)$ and replacing $(w, \theta)$ by $\left(w^{a}, \theta^{a}\right)$ in Eq. 74 :

$$
\begin{aligned}
& \left(w^{F E 1}, \theta^{F E 1}\right) \in V^{1} \text { and: } \\
& \forall\left(w^{*}, \theta^{*}\right) \in V^{1}, a\left(\left(w^{F E 1}, \theta^{F E 1}\right),\left(w^{*}, \theta^{*}\right)\right)=L\left(w^{*}, \theta^{*}\right) \\
& +F_{0}^{F E 1} w_{0}^{*}+M_{0}^{F E 1} \theta_{0}^{*}+F_{1}^{F E 1} w_{1}^{*}+M_{1}^{F E 1} \theta_{1}^{*} .
\end{aligned}
$$

According to the boundary conditions imposed to the kinematics $w_{0}^{F E 1}, \theta_{0}^{F E 1}, w_{1}^{F E 1}, \theta_{1}^{F E 1}$ and the loads $F_{0}^{F E 1}, M_{0}^{F E 1}, F_{1}^{F E 1}$, $M_{1}^{F E 1}$, some of these quantities are given and some are unknown. For instance, for the case of a cantilever beam submitted to a tip force $F$, we have $w_{0}^{F E 1}=0, \theta_{0}^{F E 1}=0, F_{1}^{F E 1}=F$ and $M_{1}^{F E 1}=0$ and the remaining quantities $w_{1}^{F E 1}, \theta_{1}^{F E 1}, F_{0}^{F E 1}$ and $M_{0}^{F E 1}$ are unknowns and a priori different from the the exact quantities $w_{1}, \theta_{1}, F_{0}$ and $M_{0}$.

In order to prove that those unknown boundary quantities are in fact the exact ones, we split the exact solution $(w, \theta)$ into :

$$
(w, \theta)=\left(w^{L}, \theta^{L}\right)+\left(w^{U}, \theta^{U}\right)
$$

where the pair $\left(w^{L}, \theta^{L}\right)$ verifies Eqs. 3 and 4 with the given distributed loadings $p$ and $m$ and is such that $w_{0}^{L}=w_{1}^{L}=0$ and $\theta_{0}^{L}=\theta_{1}^{L}=0$. Due to the linearity of the problem, the pair $\left(w^{U}, \theta^{U}\right)$ verifies Eqs. 3 and 4 with no distributed loadings and is such that $w_{0}^{U}=w_{0}, \theta_{0}^{U}=\theta_{0}, w_{1}^{U}=w_{1}$ and $\theta_{1}^{U}=\theta_{1}$.

Using this decomposition, the weak formulation (Eq. 74) reads:

$$
\begin{aligned}
& \forall\left(w^{*}, \theta^{*}\right), a\left(\left(w^{L}, \theta^{L}\right),\left(w^{*}, \theta^{*}\right)\right)+a\left(\left(w^{U}, \theta^{U}\right),\left(w^{*}, \theta^{*}\right)\right) \\
& =L\left(w^{*}, \theta^{*}\right)+F_{0} w_{0}^{*}+M_{0} \theta_{0}^{*}+F_{1} w_{1}^{*}+M_{1} \theta_{1}^{*} .
\end{aligned}
$$

Now, let's consider in the previous variational equation a virtual field $\left(w^{*}, \theta^{*}\right)$ belonging to $V^{1}$, that is to say satisfying Eqs. 3 and 4 with no distributed loadings, and let $F_{0}^{*}, M_{0}^{*}, F_{1}^{*}$ and $M_{1}^{*}$ denoting the corresponding boundary loads. As $\left(w^{*}, \theta^{*}\right)$ verifies Eqs. 3 and 4 with no distributed loadings, it satisfies the weak formulation (Eq. 9) with $L \equiv 0$ that reads:

$$
\begin{aligned}
& \forall\left(w^{* *}, \theta^{* *}\right), a\left(\left(w^{*}, \theta^{*}\right),\left(w^{* *}, \theta^{* *}\right)\right) \\
& =F_{0}^{*} w_{0}^{* *}+M_{0}^{*} \theta_{0}^{* *}+F_{1}^{*} w_{1}^{* *}+M_{1}^{*} \theta_{1}^{* *},
\end{aligned}
$$

so, considering $\left(w^{* *}, \theta^{* *}\right)=\left(w^{L}, \theta^{L}\right)$ which is such that $w_{0}^{L}=$ $w_{1}^{L}=0$ and $\theta_{0}^{L}=\theta_{1}^{L}=0$, we get:

$$
a\left(\left(w^{L}, \theta^{L}\right),\left(w^{*}, \theta^{*}\right)\right)=a\left(\left(w^{*}, \theta^{*}\right),\left(w^{L}, \theta^{L}\right)\right)=0,
$$

and the formulation in Eq. 76 reads:

$$
\begin{aligned}
& \left(w^{U}, \theta^{U}\right) \in V^{1} \text { and: } \\
& \forall\left(w^{*}, \theta^{*}\right) \in V^{1}, a\left(\left(w^{U}, \theta^{U}\right),\left(w^{*}, \theta^{*}\right)\right)=L\left(w^{*}, \theta^{*}\right) \\
& +F_{0} w_{0}^{*}+M_{0} \theta_{0}^{*}+F_{1} w_{1}^{*}+M_{1} \theta_{1}^{*},
\end{aligned}
$$

which is exactly of the same form as the weak formulation (Eq. 75) satisfied by the finite element approximation $\left(w^{F E 1}, \theta^{F E 1}\right)$ of $(w, \theta)$. That means that for any consistent boundary conditions with enough kinematic conditions to assure the uniqueness of the solution $(w, \theta)$, the pairs $\left(w^{U}, \theta^{U}\right)$ and $\left(w^{F E 1}, \theta^{F E 1}\right)$, subjected to the same boundary conditions, are identical. Therefore, for any distributed loads $p$ and $m$ and for any consistent boundary conditions imposed to $(w, \theta)$ - which are also imposed to $\left(w^{F E 1}, \theta^{F E 1}\right)$ - the imposed and unknown boundary quantities $w_{0}^{F E 1}, \theta_{0}^{F E 1}, w_{1}^{F E 1}, \theta_{1}^{F E 1}, F_{0}^{F E 1}, M_{0}^{F E 1}, F_{1}^{F E 1}$, $M_{1}^{F E 1}$ are in fact equal to the exact ones.

\section{Case studies}

In the following, we study the performance of the FCQ Timoshenko beam for linear (a cantilever beam subjected to complex distributed vertical loads or a constant distributed vertical load and a linear distributed moment) and a non linear problem (a reinforced concrete column submitted to a cyclic load).

\subsection{A cantilever Timoshenko beam subjected to complex ver- ticals loads}

Let's consider a homogeneous cantilever beam submitted to a distributed load $p(x)=q x^{n}$, to a force $F_{1}$ and a zero moment 
at its free end. According to the analytical solutions presented in Eqs. $14 \mathrm{a}$ and $14 \mathrm{~b}$, the transverse displacement and rotation at the free end take the following form:

$$
\begin{aligned}
w_{1} & =\left(e+\frac{1}{3}\right) F_{1}+\frac{q}{(n+1)(n+2)(n+3)(n+4)} \\
& -\frac{e q}{(n+1)(n+2)}-\frac{q}{2(n+1)(n+2)}+\frac{\left(e+\frac{1}{3}\right) q}{n+1}, \\
\theta_{1} & =-q \frac{1}{2(n+3)}-\frac{1}{2} F_{1} .
\end{aligned}
$$

If only one FCQ Timoshenko element is used for the spatial discretisation, the stiffness and reduced stiffness matrices of the problem are respectively given by Eqs. 40 and 64 considering $\ell_{n}=1$. The load vector and the reduced load vector become:

$$
\begin{aligned}
& \underline{\mathbf{L}}_{1}=\frac{q}{D}\left(\begin{array}{c}
6(n+2) \\
0 \\
4(n+1) \\
0 \\
-2(n+1)(n+2) \\
(n+1)(n+2)(n+6) \\
0
\end{array}\right) \\
& \underline{\mathbf{L}}_{1}^{\text {red }}=\frac{q}{(1+12 e) D}\left(\begin{array}{c}
6(n+2)+12 e(n+3)(n+4) \\
-(n+1)(2+6 e(n+4)) \\
(n+1)[(n+2)(n+6)+12 e(n+3)(n+4)] \\
(n+1)(n+2+6 e(n+4))
\end{array}\right) \text {, }
\end{aligned}
$$

where

$$
D=(n+1)(n+2)(n+3)(n+4) \text {. }
$$

The unknowns are the displacement $w_{1}$ and the rotation $\theta_{1}$ at the tip end and the reaction force $F_{0}$ and moment $M_{0}$ at the origin. The linear system satisfied by these unknowns reads:

$$
\begin{aligned}
& 2\left(\begin{array}{cccc}
6 & -3 & -6 & -3 \\
-3 & 6 e+2 & 3 & -6 e+1 \\
-6 & 3 & 6 & 3 \\
-3 & -6 e+1 & 3 & 6 e+2
\end{array}\right)\left(\begin{array}{l}
0 \\
0 \\
w_{1} \\
\theta_{1}
\end{array}\right)= \\
& \frac{q}{D}\left\{\begin{array}{c}
6(n+2)+12 e(n+3)(n+4) \\
-(n+1)(2+6 e(n+4)) \\
(n+1)[(n+2)(n+6)+12 e(n+3)(n+4)] \\
(n+1)(n+2+6 e(n+4)) \\
F_{0} \\
M_{0} \\
F_{1} \\
0
\end{array}\right)
\end{aligned}
$$

Solving the previous system one finds the analytical solutions of Eqs. 79 and 80. As proven in section 9, one FCQ Timoshenko element is able to predict the exact tip displacements for any complex distributed loadings and any suitable boundary conditions.

\subsection{A cantilever Timoshenko beam subjected to a constant} distributed vertical load and a linear distributed moment

Let's consider a homogeneous cantilever beam submitted to a constant distributed load equal to $1 \mathrm{~N} / \mathrm{m}$ and a linear distributed bending moment equal to $\hat{x} \mathrm{Nm} / \mathrm{m}$. The other parameters are: length $l=1 \mathrm{~m}$, cross-section area $A=1 \mathrm{~m}^{2}$, moment of inertia $I=1 / 12 m^{4}$, Young modulus $E=1 P a$, Poison coefficient $v=0$, shear coefficient $\kappa=1$.

Two numerical calculations are performed hereafter using respectively one and two FCQ Timoshenko elements for the spatial discretisation. Results are compared with the analytical solutions of Eqs. 4, 4a, 4b, 5 and 14. Figures 5, 6 present the distributions of the displacements and rotations along the axis of the beam and figures 7 and 8 the distributions of the moments and shear forces.

As discussed before, one FCQ Timoshenko beam reproduces the exact tip displacements. By increasing the number of FCQ 


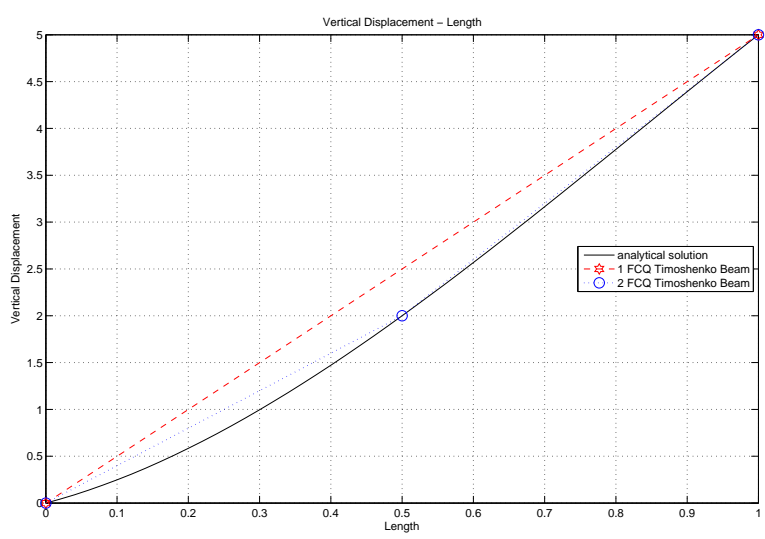

Figure 5: Transverse displacement Vs. Length.

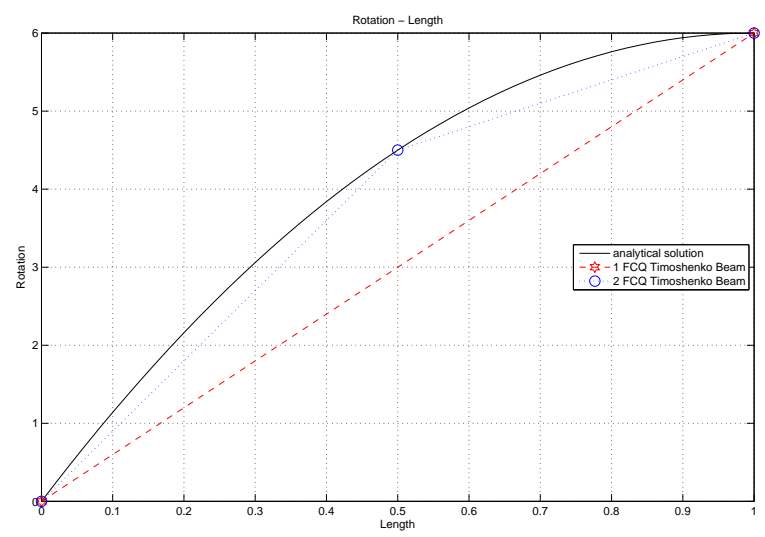

Figure 6: Rotation Vs. Length.

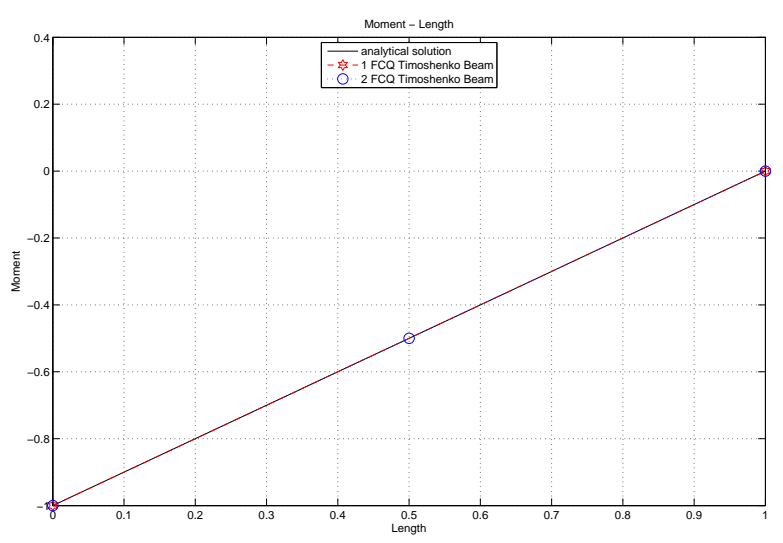

Figure 7: Moment Vs.Length.

elements results are improving within the beam. Similar numerical results and conclusions are found using the finite element proposed by Friedman and Kosmatka [11].

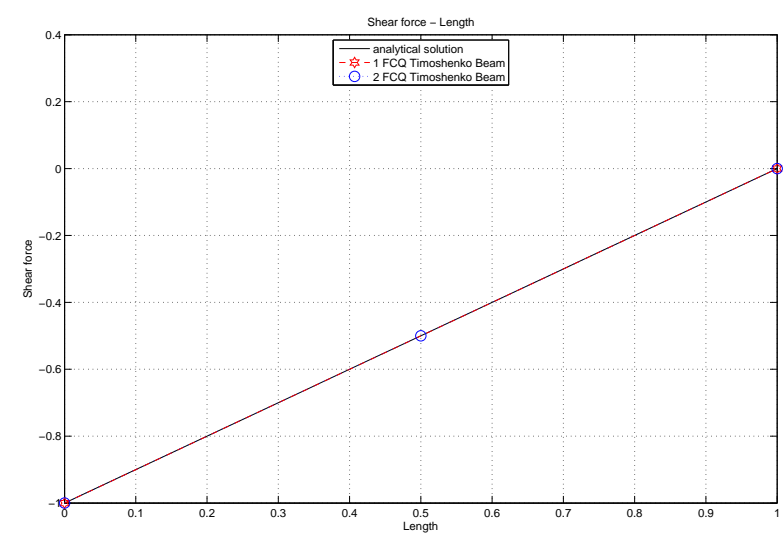

Figure 8: Shear Vs. Length.

\subsection{A reinforced concrete column submitted to a cyclic load}

In order to validate the performance of the FCQ Timoshenko beam element for a practical engineering problem, we simulate hereafter the inelastic behaviour of a reinforced concrete $(\mathrm{R} / \mathrm{C})$ column under a general three dimensional load history. The column was tested in the laboratory ELSA of the Joint Research Center (J.R.C.) in Italy [2]. The specimen has a $0.25 m \times 0.25 m$ square cross section, a free length of $1.5 \mathrm{~m}$ and is considered fixed at the base. Longitudinal reinforcement consists of eight $16 \mathrm{~mm}$ diameter bars uniformly distributed around the perimeter of the section. The concrete cover of the stirrups is $15 \mathrm{~mm}$ thick, (see Fig. 9). Reinforcement bars showed yield stress and ultimate strength of $460 \mathrm{MPa}$ and $710 \mathrm{MPa}$ respectively, the latter at a uniform elongation of $11 \%$.
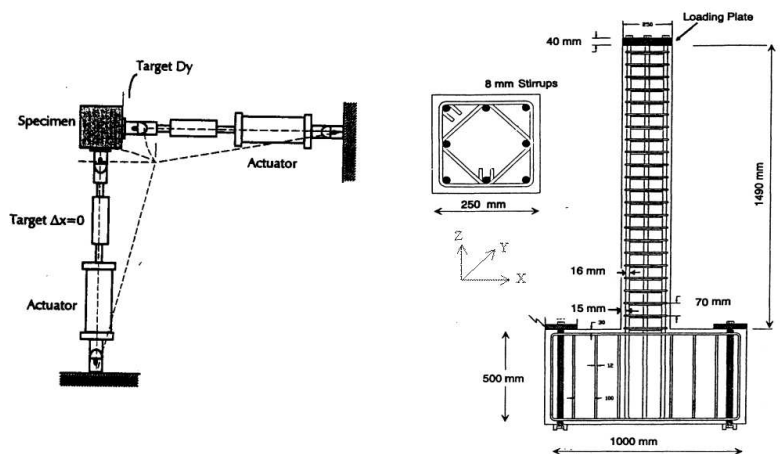

Figure 9: R/C column: description of the specimen and the experimental setup [2].

The test $\mathrm{S} 1$ of the experimental campaign is simulated here- 
after. For this test, uniaxial displacement cycles in pairs of linearly increasing amplitude are alternately applied in the two transverse directions ( $X$ and $Y$ ) at the top of the column, (see Fig. 10). A constant axial force of $0.21 M N$ is also applied during the test, through the center of a loading plate at the top of the column with an actuator located inside a steel-cup-shaped chamber.

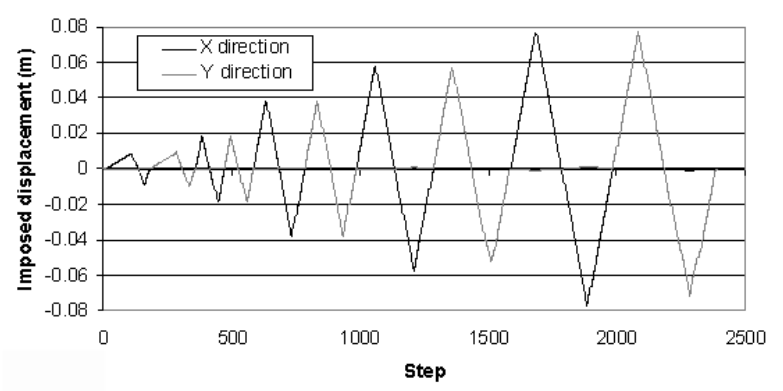

Figure 10: R/C column: displacement loading history.

Calculations are performed with the finite element toolbox FEDEASLab [10], where a new multifiber Timoshenko beam has been implemented based on the FCQ element (the reader can find more information about the multifiber beam technology in [12], [14], [17], [22]). Five FCQ multifiber Timoshenko beam elements are used to model the R/C column. Each section has 16 fibers for concrete and 8 fibers for steel. The base slab is not simulated and the specimen is considered fixed at the base. 1D constitutive laws are adopted for concrete [15] and steel [18] based on damage mechanics and plasticity respectively. Shear and torsion are considered linear and the shear coefficient $\kappa$ is taken constant. Confinement effects are not taken into account.

Comparison of the numerical and experimental results for both directions is represented in Figs. 11 and 12. The model simulates correctly the global behaviour of the mock-up in terms of displacements and forces. Calculation is not time consuming allowing for parametrical studies.

\section{Conclusions}

The formulation of a Timoshenko finite element beam with internal degrees of freedom, suitable for material non linearity

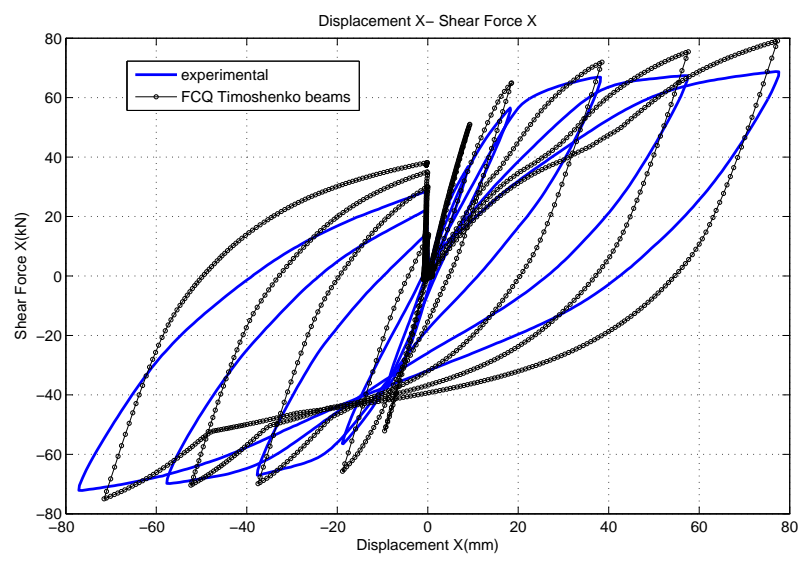

Figure 11: R/C Column: numerical vs experimental results in the $\mathrm{X}$ direction.

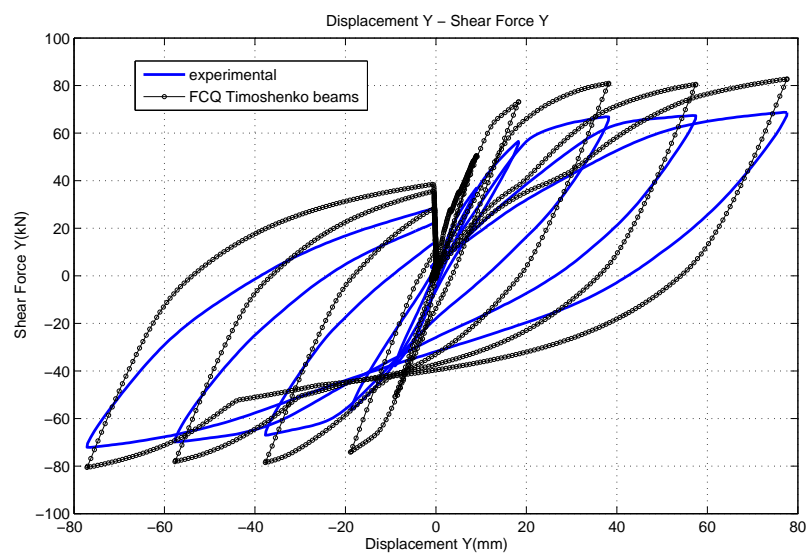

Figure 12: R/C Column: numerical vs experimental results in the $\mathrm{Y}$ direction.

problems, has been presented in this paper (FCQ Timoshenko beam). Cubic shape functions are used for the transverse displacements and quadratic for the rotations. The element is free of shear locking and one element is able to predict the exact tip displacements for any complex distributed loadings and any suitable boundary conditions. One element gives the exact solution of the bending of a Timoshenko beam free of distributed loadings or for $p=0$ and $m$ constant. Being a displacement based formulation element, it can be introduced into any general purpose finite element code without any particular modification in its architecture.

We have also proven that the element presented in [11], with shape functions depending on material properties, derive from the FCQ element. Numerical results show the performance 
of the FCQ Timoshenko finite element beam under static and cyclic loadings, for elastic but also non elastic material behaviour (FCQ multifiber Timoshenko beam). Detailed comparisons in terms of accuracy and robustness with other Timoshenko beam formulations can be found in [1].

\section{Acknowledgements}

The authors would like to thank Pr. Bousias S. from the University of Patras (Greece) for providing the experimental results of the reinforced concrete column.

\section{References}

[1] I. Bitar, S. Grange, P. Kotronis, N. Benkemoun, A review on Timoshenko multifiber beams finite element formulations and elasto-plastic applications, Engineering Structures (2015) (submitted).

[2] S.N. Bousias, G. Verzeletti, M.N. Fardis, E. Guiterrez, Load-path effects in column biaxial bending and axial force, J. Eng. Mech. ASCE 121 (5) (1995) 596-605.

[3] D. Caillerie, Étude de la convergence et du verrouillage des schémas éléments finis. Poutre de Navier Bernoulli et Timoshenko, Communication privée, Laboratoire Sols, Solides, Structures-Risques, INP/UJF/CNRS, (2008).

[4] G. Casaux, Modélisation tridimensionnelle du comportement sismique d'ouvrages en béton armé - Développement de méthodes simplifiées, $\mathrm{PhD}$ ENS de Cachan, (2003).

[5] Code Aster. http://www.code-aster.org

[6] R.D. Cook, D.S. Malkus and M.E. Plesha, Concepts and Applications of Finite Element Analysis, J. Wiley, third edition, (1989).

[7] G.R. Cowper, The shear coefficient in Timoshenko's beam theory, Journal of Applied Mechanics, Transactions of the ASME, 33 (1966) 335-340.

[8] R. Cybulski, Numerical issues on beam finite elements, Report Master MEMS-GGCR, Laboratoire Sols, Solides, Structures-Risques, UJF/INP/CNRS, (2008).

[9] V. De Ville de Goyet, L'analyse statique non linéaire par la méthode des éléments finis des structures spatiales formées de poutres à section non symétrique. Thèse de doctorat à l'Université de Liége, (1989).

[10] F.C. Filippou, M. Constandines, FedeasLab Getting Started Guide And Simulations Examples, Department of civil and Environmental Engineering UC Berkeley, (2004), http://www.ce.berkeley.edu/ filippou/FEDEASLab/FEDEASLab.htm

[11] Z. Friedman, Z.B. Kosmatka, An improved two-node Timoshenko beam finite element, Computers and structures, 47 (3) (1993) 473-481.
[12] J. Guedes, P. Pégon, A. Pinto, A fibre Timoshenko beam element in CASTEM 2000. Special publication Nr. I.94.31., J.R.C., I-21020, Ispra, Italy, (1994).

[13] A. Ibrahimbegovic, F. Frey, Finite element analysis of linear and non linear deformations of elastic initially curved beams. LSC internal report 92/02, January, Department of Civil Engineering, Swiss Federal Institute of Technology, LSC, DGC,EPFL, Lausanne, (1992).

[14] P. Kotronis, J. Mazars, Simplified modelling strategies to simulate the dynamic behaviour of R/C walls, Journal of Earthquake Engineering, 9 (2) (2005) 285-306.

[15] C.L. La Borderie, Phénomènes unilatéraux dans un matériau endommageable: modélisation et application á l'analyse des structures en béton, PhD Université Paris 6, (1991).

[16] Maxima. http://maxima.sourceforge.net

[17] J. Mazars, P. Kotronis, F. Ragueneau, G. Casaux, Using multifiber beams to account for shear and torsion. Applications to concrete structural elements, Computer Methods in Applied Mechanics and Engineering, 195 (52) (2006) 7264-7281.

[18] M. Menegoto, P. Pinto, Method of analysis of cyclically loaded reinforced concrete plane frames including changes in geometry and non-elastic behaviour of elements under combined normal force and bending, IABSE Symposium on resistance and ultimate deformability of structures acted on by well-defined repeated loads, final report, Lisbon, 328p, (1973).

[19] R.E. Nickel, G.A. Secor, Convergence of consistently derived Timoshenko beam finite elements, International Journal for Numerical Methods in Engineering, 5 (1972) 243-253.

[20] P. Pegon, A Timoshenko simple beam element in CASTEM 2000, special publication Nr. I.94.04, JRC, I-21020 Ispra, Italy, (1994).

[21] J.S. Przemieneicki, Theory of matrix structural analysis, McGraw-Hill, New York, 70-82, (1968).

[22] E. Spacone, F.C. Filippou, F.F. Taucer, Fiber Beam-Column Model for Nonlinear Analysis of R/C Frames. I: Formulation, Earthquake Engineering and Structural Dynamics, 25(7) (1996) 711-725.

[23] A. Tessler, S.B. Dong, On a hierarchy of conforming Timoshenko beam elements, Computers and Structures, 14(3-4) (1981) 335-344.

[24] D.L. Thomas, J.M. Wilson, R.R. Wilson, Timoshenko beam finite elements, Journal of Sound and Vibration, 31(3) (1973) 315-330.

[25] J. Thomas, B.A.H. Abbas, Finite element model for dynamic analysis of Timoshenko beam, Journal of Sound and Vibration, 41(3) (1975) 291299. 